\title{
Stereotactic Body Radiation Therapy in Primary and Metastatic Liver Disease
}

\author{
DONATELLA CAIVANO ${ }^{1}$, MAURIZIO VALERIANI ${ }^{2}$, IVANA RUSSO ${ }^{2}$, PAOLO BONOME $^{2}$, \\ SARA DE MATTEIS ${ }^{2}$, GIUSEPPE MINNITI ${ }^{3}$ and MATTIA FALCHETTO OSTI ${ }^{2}$ \\ ${ }^{1}$ CNAO, National Centre of Oncological Hadrontherapy, Pavia, Italy; \\ ${ }^{2}$ Department of Radiation Oncology, University of Rome "Sapienza", \\ Faculty of "Medicina e Psicologia", Sant'Andrea Hospital, Rome, Italy; \\ ${ }^{3}$ Neuromed - Mediterranean Neurological Institute, Pozzilli, Italy
}

\begin{abstract}
Background/Aim: The aim of this study was to investigate the treatment outcomes and toxicities in patients with liver disease treated by Stereotactic Body Radiation Therapy (SBRT). Patients and Methods: From 2007 to 2016, 43 patients with 58 lesions (6 primary and 37 metastatic liver tumors) were treated with SBRT. Results: Local Control was reached in 47 out of $58(81 \%)$ treated lesions with 12 and 24-month rates of $81 \%$ and $74 \%$ respectively. The progression-free survival at 12 and 24 months was $42 \%$ and $36 \%$, respectively. The disease specific survival at 12 and 24 months was $74 \%$ and $46 \%$ respectively. Median overall survival (OS) was 20 months and the rates of OS were $74 \%$ and $46 \%$ at 12 and 24 months respectively. Toxicity was very low consisting mainly of Grade 1 and 2. Conclusion: SBRT provides good local control for both primary and metastatic liver lesions, with minimal toxicity.
\end{abstract}

The liver is a common site of metastases that sometimes occur at the beginning of disease, in these situations a premature diagnosis might allow for an early intervention that might delay progression. For primary and isolated metastatic liver tumors resection and systemic therapy are associated with better overall survival, but only $10-25 \%$ is resectable. At the moment the curative management of unresectable hepatic tumors represents a major task. There are other ablative treatments, such as microwave hyperthermia, radiofrequency ablation (RFA), cryosurgery, ethanol injection, transarterial chemoembolization and Stereotactic Body Radiation Therapy (SBRT) (1-2). The effectiveness of the standard radiotherapy

Correspondence to: Dr. Donatella Caivano, CNAO, National Centre of Oncological Hadrontherapy, Strada Privata Campeggi 55, 27100, Pavia, Italy. Tel: +39 3407646521, e-mail: donatella.caivano@gmail.com

Key Words: SBRT, toxicities, outcomes of survival, local control, univariate analysis is based on the damage of the double-stranded DNA which causes progressive cell death; however, SBRT may associate with additional lesions such as vascular lesions, resulting in an increased effect, more ablative. The relationship between radiation dose and cell death has historically been modeled using Linear-quadratic model. Current data suggest how this model would underestimate the rate of cell damage after the use of stereotactic radiotherapy (3). To avoid these contradictory data, we need more rigorous studies about radiobiology in the SBRT. Over the past decade, several large prospective Phase 1 and 2 trials have shown that the use of SBRT for the management of primary tumors in the liver and in liver metastasis is able to provide good results in terms of Local Control. The SBRT has proven to be non-invasive, safe and with minimal toxicity. The SBRT is a technique able to deliver an ablative dose of radiation to the target with 5 or fewer fractions, saving surrounding healthy parenchyma of liver and organs at risk (OARs). Furthermore, several studies have shown that the results in terms of Local Control and Overall Survival can change with respect to the number of metastases, size, histology, dose and systemic therapy associated (4-7). We report our experience of patients treated in our center to evaluate Local Control (LC), ProgressionFree Survival (PFS), Disease Specific Survival (DSS) and Overall Survival (OS). Moreover, we correlate survivals with dose, histology, size, synchronous and metachronous metastases, extra-hepatic disease and chemotherapy post treatment and monitoring acute and late toxicities.

\section{Materials and Methods}

Patients. From December 2007 to March 2016, 43 patients with 58 lesions in the liver not eligible for surgical resection due to local tumor extension or patients comorbidities or patients who refuse surgery were included in this study. The median age at time of enrollment was 69 years (range $=37-86$ years). There were 22 female $(51 \%)$ and 11 male (49\%). Metastatic tumors comprised the majority of patients $(86 \%)$, with Gastro-Intestinal Stromal Tumor (GIST) 
(2\%), colorectal (CRC) (35\%), endometrial (2\%), gastric $(7 \%)$, breast (17\%), lung (14\%), pancreatic (7\%), Small Cell Lung Cancer (SCLC) (2\%) primary. Hepatocellular carcinoma (HCC) and intrahepatic cholangio-carcinoma represented the $5 \%$ and $9 \%$ of all patients, respectively. Thirty-six patients $(84 \%)$ received chemotherapy before or after SBRT and 7 patients (16\%) did not receive chemotherapy. Patient characteristics are presented in Table I. Patients with 1-3 liver lesions were considered eligible after discussion in a multidisciplinary tumor-board. Other inclusion criteria were an Eastern Cooperative Oncology Group (ECOG) PS $\leq 2$, lesion size $<200 \mathrm{cc}$ and a diameter $<7,5 \mathrm{~cm}$. Patients were included even if their disease was not confined to the liver. The number of treated lesions ranged from one to three, thirty-three patients (76\%) had 1 single lesion, 5 patients (12\%) had 2 lesions, 5 patients $(12 \%)$ had 3 lesions. Pretreatment investigations in all patients consisted of physical examination, laboratory tests including blood counts and liver enzymes, computed tomography (CT) scan of the thorax and abdomen with i.v. contrast and - in selected patients - magnetic resonance imaging (MRI) and wholebody positron emission tomography (PET, Tracer: 18-F-fluorodeoxyglucose (FDG)) on a dedicated combined PET/CT hybrid scanner. Written informed consent was obtained before beginning radiation therapy.

SBRT planning and delivery. All patients underwent computed tomography (CT) simulation and were immobilized in the supine position. The 4DCT was used to generate an internal target volume. When the target lesion was not readily apparent on the CT images, the planning data set was registered to a pretreatment diagnostic MRI or $\mathrm{PET} / \mathrm{CT}$, using a mutual information algorithm in our in-house treatment planning system, to facilitate target delineation (8). The Gross Tumor Volume (GTV) and the Internal Target Volume for free-breathing cases were expanded by a 4-mm radial and 4-mm cranio-caudal margin for the Planning Target Volume (PTV). SBRT was planned and delivered using three-dimensional conformal techniques with multiple (typically $\geq 5$ and $\leq 9$ ), non-opposed, coplanar static X 6-15 MV beams. Radiation dose was prescribed to the isodose surface covering the $95 \%$ of the maximum PTV dose. Forty lesions (69\%) were treated with one fraction: 20 Gy in 1 case, 23 Gy in 20 cases and 30 Gy in 19 cases. Fifteen lesions (26\%) were treated with 3 fractions: total dose $42 \mathrm{~Gy}$ in 1 case, 45 Gy in 10 cases, 54 Gy in 4 cases. Three lesions (5\%) were treated with 5 fractions: total dose of 40,38 and $30 \mathrm{~Gy}$, respectively. Generally, multi-fractions were used for lesions near organs at risk and great vessels. The biologically effective dose (BED) $(\alpha / \beta=10 \mathrm{~Gy})$ was 48-151.2 Gy (median=112.5Gy). The BED10 formula used is: BED $(\mathrm{Gy} 10)=$ nd $(1+\mathrm{d} / \alpha / \beta)$. Dose limits to the Organs at risk are presented in Table II. Daily image guidance and positioning was performed with cone beam CT.

Follow-up. After treatment, patients were followed up with physical examination, tumor markers, serum liver enzymes, and imaging, alternating CT, MRI, or positron emission tomography (PET). Patients were evaluated after 2-3 months for the first 2 years and then every 6 months. Follow up imaging was reviewed by a radiation oncologist. Treatment response was assessed for each treated lesion and scored using the Response Evaluation Criteria in Solid Tumors (RECIST) (6). Local recurrence was scored as in-field or marginal failure. Toxicity was evaluated with the CTCAEv.4 scale. Acute toxicity was defined as adverse events occurring within three months after SBRT. Late toxicity was defined as that occurring after three months to the last follow-up.
Table I. Patient and tumor characteristics.

\begin{tabular}{|c|c|c|}
\hline & No. & $\%$ \\
\hline Total no. of patients & 43 & \\
\hline Total no. of lesions evaluated & 58 & \\
\hline \multicolumn{3}{|l|}{ Age, years } \\
\hline Median & 69 & \\
\hline Range & $37-86$ & \\
\hline Primary liver tumors & & 14 \\
\hline Hepatocellular carcinoma & 2 & 5 \\
\hline Intrahepatic cholangiocarcinoma & 4 & 9 \\
\hline Metastatic liver tumors & & 86 \\
\hline Gastro-Intestinal Stromal Tumor(GIST) & 1 & 2 \\
\hline Colorectal primary & 15 & 35 \\
\hline Endometrial primary & 1 & 2 \\
\hline Gastric primary & 3 & 7 \\
\hline Breast primary & 7 & 17 \\
\hline Lung & 6 & 14 \\
\hline Pancreatic & 3 & 7 \\
\hline SCLC & 1 & 2 \\
\hline \multicolumn{3}{|l|}{ Lesion volume (CM3) } \\
\hline Medium & 32,73 & \\
\hline Range & 2-194.5 & \\
\hline \multicolumn{3}{|l|}{ Performance status (PS) } \\
\hline 0 & 21 & 49 \\
\hline 1 & 21 & 49 \\
\hline 2 & 1 & 2 \\
\hline \multicolumn{3}{|l|}{ Median biologic equivalent dose (BED10) } \\
\hline$<100$ & 24 & 41 \\
\hline$\geq 100$ & 34 & 58 \\
\hline \multicolumn{3}{|l|}{ Chemotherapy post SBRT } \\
\hline Yes & 42 & 79 \\
\hline No & 11 & 21 \\
\hline \multicolumn{3}{|l|}{ Extrahepatic disease } \\
\hline Yes & 26 & 49 \\
\hline No & 27 & 51 \\
\hline
\end{tabular}

Table II. Dosimetric constraints.

\begin{tabular}{lc}
\hline Stomac & $\mathrm{D} \max <30 \mathrm{~Gy}$ \\
Bowel & $\mathrm{D} \max <30 \mathrm{~Gy}$ \\
Spinal cord & $\mathrm{D} \max \leq 20 \mathrm{~Gy}$ \\
Healthy liver & $>700 \mathrm{~cm}^{3}, 15 \mathrm{~Gy}$ \\
\hline
\end{tabular}

Evaluation of response and statistical analysis. The primary end point was LC, defined as the absence of new or progressive lesions within or at the margin of the PTV. Secondary end points were PFS, DSS, OS and toxicities. PFS was calculated from the first day of SBRT and was defined as freedom from any local, distant intrahepatic or distant extra hepatic progression. DSS was calculated from the first day of treatment to the death for disease; patients deceased from causes not related to the disease were not considered as an event. OS was calculated from the start of SBRT until death. LC, PFS, DSS and OS were estimated using the Kaplan-Meier method. Differences between survival curves were tested with log- 


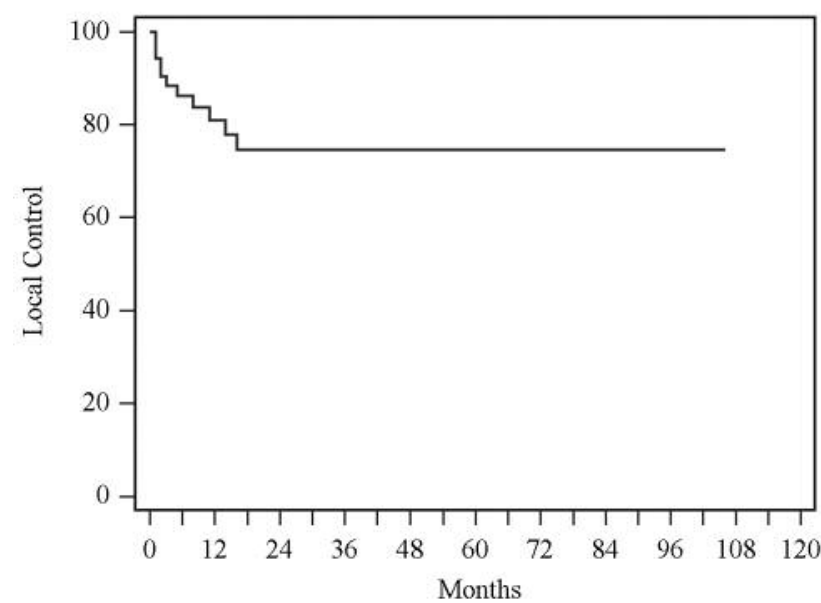

Figure 1. Kaplan Meier curves showing local control.

rank test analysis. All statistical analyses were performed using the software package SPSS, version 22 (IBM corporation). A $p \leq 0.05$ was considered statistical significant.

\section{Results}

Median follow-up time was 16 months for all patients (range $=1-106$ months) and 24 months (range=8-106 months) for living patients.

Clinical outcome. Local Control Local Control was reached in 47 out of $58(81 \%)$ treated lesions, with 12 and 24- months rates of $81 \%$ and $74 \%$ respectively (Figure 1). We have not found prognostic factors for local control in univariate analysis for histology (colorectal cancer vs. other primary cancers $v s$. primary liver tumor, $p=0.528$; metastatic lesions $v s$. primary lesions, $p=0.267$ ), dose (BED $10 \geq 100 v s$. BED $10<100, p=0.752$ ), size (diameter $\geq 3 \mathrm{~cm} v s$. diameter $<3$ $\mathrm{cm}, p=0.499$; medium volume $\geq 33 \mathrm{cc} v s .<33 \mathrm{cc}, p=0.490$ ), synchronous $v s$. metachronous metastasis and LC $(p=0.239)$, extrahepatic disease (extrahepatic lesions $v s$. disease confined to the liver, $p=0.882$ ) and chemotherapy (chemotherapy post treatment $v s$. not chemotherapy, $p=0.730)$.

Progression free survival. The PFS at 12 and 24-months was $42 \%$ and $36 \%$ respectively (Figure 2). The median time to progression was 8 months (95\% CI=5-11 months). Thirty (70\%) patients developed progression of disease. In the univariate analysis we found that histology (colorectal cancer $v s$. other primary cancers $v s$. primary liver tumor, $p=0.866$; metastatic lesions $v s$. primary lesions, $p=0.911$ ), dose (BED10 $\geq 100 v s$. BED10<100, $p=0.938$ ), size (diameter $\geq 3 \mathrm{~cm} v s$. diameter $<3 \mathrm{~cm}, p=0.152$; medium volume $\geq 33 \mathrm{cc} v s$. $<33 \mathrm{cc}, p=0.916$ ), synchronous $v s$. metachronous, $p=0.415$, extrahepatic disease

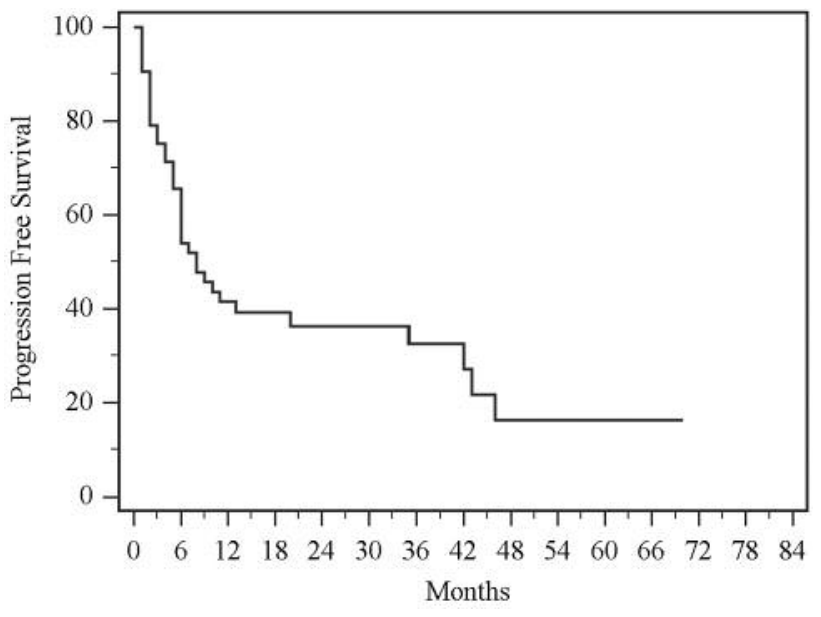

Figure 2. Kaplan Meier curves showing progression free survival.

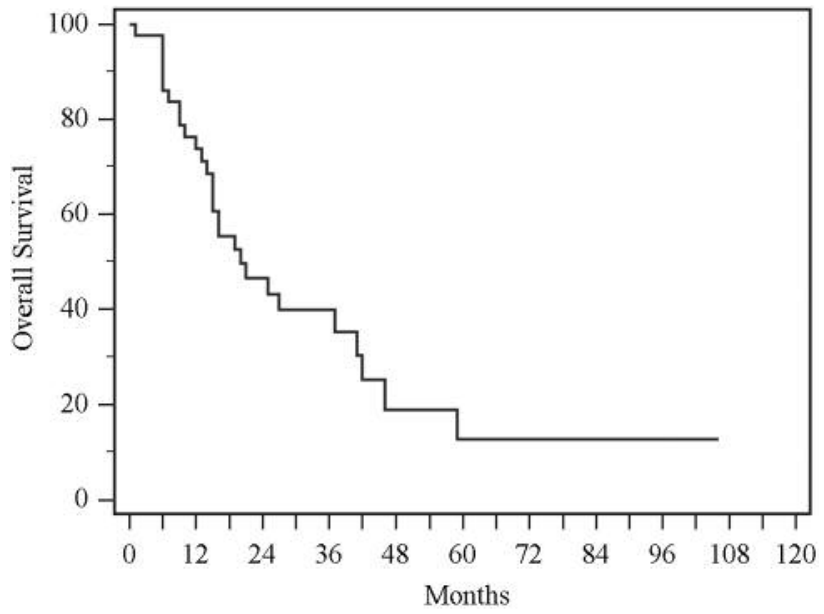

Figure 3. Kaplan Meier curves showing overall survival.

(extrahepatic lesions $v s$. disease confined to the liver, $p=0.855$ ) are not significantly correlated with progression of disease. However, there is a statistical correlation with chemotherapy (not chemotherapy post treatment SBRT $v s$. chemotherapy, $p=0.037)$.

Disease specific survival. The Disease Specific Survival at 12 and 24 -months was $74 \%$ and $46 \%$ respectively. We did not observe differences with OS because only one patient deceased from other reasons. The median time of death from cancer was 20 months $(95 \% \mathrm{CI}=8-32$ months). In the univariate analysis we did not find a statistical correlation with: histology (colorectal cancer $v s$. other primary cancers $v s$. primary liver tumor, $p=0.244)$, dose (BED10 $\geq 100 v s$. $\mathrm{BED} 10<100, p=0.146$ ), size (diameter $\geq 3 \mathrm{~cm} v s$. diameter $<3$ 
$\mathrm{cm}, p=0.977$; medium volume $\geq 33 \mathrm{cc} v s .<33 \mathrm{cc}, p=0.663$ ) and synchronous $v s$. metachronous metastasis, $p=0.823$.

Overall survival. Median overall survival was 20 months (95\% CI=8-32 months), with 12 and 24-months rates of $74 \%$ and $46 \%$, respectively (Figure 3 ). At the last follow-up twenty-eight patients $(65 \%)$ had deceased (one of these deaths was not cancer related). The statistical study did not show correlation with dose, histology, size, synchronous and metachronous metastases, extra-hepatic disease and chemotherapy post treatment.

Toxicity. The treatment was well tolerated and all patients completed therapy without treatment interruptions. Five patients $(11 \%)$ experienced acute toxicity: one patient $(2 \%)$ had Grade 1 nausea, 1 patient (2\%) had Grade 2 nausea, 2 patients $(5 \%)$ had abdominal pain Grade 1, one of this had also diarrhea Grade 1. One patient (2\%) had abdominal pain Grade 2. Late toxicities were recorded in 3 patients (7\%): two patients (5\%) experienced abdominal pain Grade 1, one of this had also sporadic diarrhea. One patient (2\%) experienced nausea Grade 2, weight loss at 4 months from SBRT requiring antiemetics, and deceased 6 months after SBRT from gastrointestinal hemorrhage, not well specified.

\section{Discussion}

Historically, one of the major limitations of utilization of radiation therapy for treatment of primary and secondary liver lesions was the Radiation Induced Liver Disease (RILD). The advances in radiation therapy have provided the possibility to reduce toxicity using SBRT (9). Liver SBRT can be delivered using either a linear accelerator (linac) or an SBRT-specific delivery platform, such as the robotic CyberKnife (Accuray) or a volumetric modulated arc therapy of RapidArc $(10,11)$. SBRT is less invasive than surgery; this implies better results in quality of life (QOL). With surgery we have observed a significant deterioration in health-related QOL for patients with liver metastases. By contrast, this did not occur after SBRT (12-14). Here we report the results of a retrospective study. We have evaluated LC, PFS, DSS and OS and the correlations with the histology, volume, dose and type of metastasis, synchronous or metachronous, extra-hepatic disease and chemotherapy post treatment with a univariate analysis. Metastatic tumors comprised the majority of patients $(86 \%)$. Hepatocellular carcinoma and intrahepatic cholangio-carcinoma represented only the $14 \%$. In our study LC was the $58 \%$, with rates of $81 \%$ and $74 \%$ at 12 and 24 months respectively and there are not correlations with the variables studied. The PFS was $42 \%$ and $36 \%$ at 12 and 24 months and OS $74 \%$ and $46 \%$ at 12 and 24-months. Surprisingly, chemotherapy was not able to change the survivals, we found a good disease free survival from SBRT without the use of chemotherapy post $(p=0.037)$. This shows that the use of chemotherapy does not change the progression of disease. In 2013 Scorsetti et al. (Phase II study) showed a LC rate for liver metastasis of $94 \%$ at 12-months, with a dose 52,5-75 Gy (3 fractions), with a medium volume of 18,6 cc (range=1.8-134 cc). In 2015 Scorsetti et al. (Phase II trial) showed also a LC of $85.8 \%$ at 12-months for HCC, with a dose of 36-75Gy (3-6 fractions) and a medium volume of 4,8cc (range=1-12.5 cc). In 2009 Kopek et al. (Phase I/II trial) obtained a LC of $85 \%$ at 12 months in the cholangio-carcinoma, whith a dose of $45 \mathrm{~Gy}$ ( 3 fractions) and a medium volume of $32 \mathrm{cc}$ (range $=9-205 \mathrm{cc}$ ) (15-17). In 2015 Andratschke et al. observed rates of LC at 12 and 24 -months of $74.7 \%$ and $48.3 \%$ respectively, for the treatment of liver metastasis by SBRT with a median dose of 35 Gy in 5 fractions. Only the minimal biologically effective dose, BED10>120 Gy to GTV $(p<0.0001)$ and tumor volume (GTV volume $<100 \mathrm{cc})(p=0.03)$ were predictive for local control. Median OS was 27 months with survival rates of $77 \%$ and $30 \%$ at 12 and 24-months. In univariate survival analysis they have observed that extrahepatic disease status $(p=0.005)$, smaller GTV volume, $<100 \mathrm{cc}(p<0.001)$ and colorectal primary $(p=0.04)$ were correlated significantly with better overall survival. In multivariate analysis only the minimal BED to the GTV remained as independent prognostic factor for LC of the irradiated lesions $(p=0.015)$ and GTV volume remained as an independent significant prognostic factor for OS ( $p=0.002)$ (5). In the study of Ahmed et al. 2016 significant differences were observed based on primary histology. There was a significant difference in LC of colorectal $v s$. non-colorectal liver metastases. The rates of LC for colorectal lesions were $79 \%$ and $59 \%$ at 12 and $24-$ months, compared with $100 \%$ for non-colorectal lesions $(p=0.019)$. There was no significant difference in OS between patients treated for colorectal and non-colorectal metastases. Other factors including dose (50 Gy vs. $60 \mathrm{~Gy}, p=0.10$ ), size ( $2<$ or $\geq 2 \mathrm{~cm}, p=0.47)$ and number of lines of previous chemotherapy $(p=0.42)$, age $(p=0.12)$, and gender $(p=0.47)$ were not found to be significant predicting factors for LC (18). The American Association for Physics in Medicine (AAPM) in an SBRT working group analyzed the relationship of LC and dose fractionation schemes for primary and metastatic liver tumor. The result was a significant better local control after the delivery of high biologically effective doses BED10 >100Gy (19). Rusthoven et al. obtained a better LC with lesions $<3 \mathrm{~cm}$ (7). However, other studies did not demonstrate this relationship $(20,21)$. In our study we did not find the same results. There is no statically significant correlation of histology, size and dose with LC, PFS and DSS. Regarding dose an explanation may be the different prescription method. In fact, the same dose prescribed to different isodoses, may result in a very different real dose delivery. In our study we prescribe at 95\% isodose. In 2016 
Takeda et al. have compared the results of LC at 3-years with a dose of 50-60 Gy (5 fractions) with a prescription at $60 \%$ isodose $v s$. a dose of 50 Gy ( 5 fractions) at $80 \%$ isodose. The rates of LC were $100 \%$ and $73 \%$ respectively. Another study showed rates of LC at 12 and 24-months of $92 \%$ and $66 \%$ respectively, with a dose of 30-37.5 (3 fractions) at 65\%isodose. They concluded that the optimal methods of prescription can change the results in terms of LC (22-25). Another possible reason for the different rates of LC is the margin from CTV to PTV. Tumor motion is present in the liver. Usually we observe a movement predominant in a cranio-caudal direction due to diaphragmatic movement during respiration. Also, the histology should be considered in the evaluation of margins. In 2000, Giraud et al. have reported that the CTV margin must be increased to $8 \mathrm{~mm}$ and $6 \mathrm{~mm}$ for adenocarcinoma and squamous cell carcinoma, respectively, to cover 95\% of the microscopic extension (26). In our study we used a 4-mm radial and 4-mm cranio-caudal margin for the PTV from the GTV and ITV. Andratschke et al. added $5 \mathrm{~mm}$ of margin in axial dimension and $10 \mathrm{~mm}$ of margin in longitudinal dimension, but in this case we observed a LC of $81 \%$ at 1 year in our study and $74,7 \%$ in the other study (5). In our study we reported poor toxicity, Grade 1 and 2. In the literature low rate of toxicity was reported. In the Phase I study by Lee et al., they observed that the risk of serious liver toxicity was low $(95 \% \mathrm{CI}=0-5.3 \%)$ (27). In 2015 Scorsetti et al. described in elderly patients who received SBRT, Grade 2-3 toxicities in five patients (4.2\%); Grade 1 toxicity in seven patients $(5.9 \%)$ and no toxicity was observed in $85.4 \%$ of all cases (28). The limits of our study are: retrospective data, low number of treated lesions and patients, and a variety of pre-SBRT and post-SBRT systemic and liver-directed therapies. Another limit of our retrospective study is that at beginning of treatments by SBRT there was no protocol at our department, so different radiation dose patterns were used, thus this can imply a statistically poor conclusion. Other limits are the multiple histological pattern and the low number of patients with primary disease, for this reason we considered it appropriate to correlate colorectal cancer $v s$. other primary cancers $v s$. primary liver tumor. Finally, in the literature there are some studies about the different responses between colorectal cancer $v s$. other primary cancers.

\section{Conclusion}

The results of our study demonstrated the effectiveness, in terms of Local Control and safety of SBRT in the treatment of primary and metastatic tumor. Prognosis remains poor with a low rate of long-term survival. Prognostic factors analyzed have not significantly impacted on the results. Further studies are in progress in order to find patients that could benefit from a more intensive treatment.

\section{References}

1 Caivano D, Bracci S, Russo I, Montalto A, Armosini V, De Sanctis V, Valeriani M, Minniti G, Enrici RM and Osti MF: Stereotactic Body Radiation Therapy for Liver Lesions. A Single-institution Experience. Anticancer Res 35: 4171-4175, 2015.

2 Kirichenko A, Gayou O, Parda D, Kudithipudi V, Tom K, Khan A, Abrams P, Szramowski M, Oliva J, Monga D, Raj M and Thai N: Stereotactic body radiotherapy (SBRT) with or without surgery for primary and metastatic liver tumors. HPB (Oxford) 18: 88-97, 2016.

3 Song CW, Cho LC, Yuan J, Dusenbery KE, Griffin RJ and Levitt SH: Radiobiology of stereotactic body radiation therapy/ stereotactic radio surgery and thelinear-quadratic model. Int J Radiat Oncol Biol Phy 87: 18-19, 2013.

4 Andratschke N, Parys A, Stadtfeld S, Wurster S, Huttenlocher S, Imhoff D, Yildirim M, Rades D, Rödel CM, Dunst J, Hildebrandt $G$ and Blanck: Clinical results of mean GTV dose optimized robotic guided SBRT for liver metastases. Radiat Oncol 11: 74, 2016.

5 Andratschke NH, Nieder C, Heppt F, Molls M and Zimmermann F: Stereotactic radiation therapy for liver metastases: factors affecting local control and survival. Radiat Oncol 10: 69, 2015.

6 Janoray G and Mornex F: Suivi après radiothérapie stéréotaxique des tumeurs hépatiques: revue de la littérature et recommandations. Cancer Radiother 19: 573-581, 2015.

7 Rusthoven KE, Kavanagh BD, Cardenes H, Stieber VW, Burri SH, Feigenberg SJ, Chidel MA, Pugh TJ, Franklin W, Kane M, Gaspar LE and Schefter TE: Multi-institutional phase I/II trial of stereotactic body radiation therapy for liver metastases. J Clin Oncol 27: 1572-1578, 2009.

8 Roberson PL, McLaughlin PW, Narayana V, Troyer S, Hixson GV and Kessler ML: Use and uncertainties of mutual information for computed tomography/ magnetic resonance (CT/MR) registration post permanent implant of the prostate. Med Phys 32: 473-482, 2005.

9 Farach A, Quesada J and Teh BS: Liver regeneration following repeat SBRT. J Gastrointest Oncol 6: E2-6, 2015.

10 Aitken KL and Hawkins MA: Stereotactic Body Radiotherapy for Liver Metastases. Clin Oncol (R Coll Radiol) 27: 307-315, 2015.

11 Paik EK, Kim MS, Choi CW, Jang WI, Lee SH, Choi SH, Kim $\mathrm{KB}$ and Lee DH: Dosimetric comparison of volumetric modulated arc therapy with robotic stereotactic radiation therapy in hepatocellular carcinoma. Radiat Oncol J 33: 233241, 2015.

12 Méndez Romero A, Wunderink W, van Os RM, Nowak PJ, Heijmen BJ, Nuyttens JJ, Brandwijk RP, Verhoef C, Ijzermans JN and Levendag PC: Quality of life after stereotactic body radiation therapy for primary and metastatic liver tumors. Int $\mathbf{J}$ Radiat Oncol Biol Phys 701: 1447-1452, 2008.

13 Klein J, Dawson LA, Jiang H, Kim J, Dinniwell R, Brierley J, Wong R, Lockwood G and Ringash J: Prospective Longitudinal Assessment of Quality of Life for Liver Cancer Patients Treated With Stereotactic Body Radiation Therapy. Int J Radiat Oncol Biol Phys 93: 16-25, 2015.

14 Langenhoff BS, Krabbe PF, Peerenboom L, Wobbes T and Ruers TJ: Quality of life after surgical treatment of colorectal liver metastases. Br J Surg 93: 1007-1014, 2006. 
15 Scorsetti M, Arcangeli S, Tozzi A, Comito T, Alongi F, Navarria P, Mancosu P, Reggiori G, Fogliata A, Torzilli G, Tomatis S and Cozzi L: Is stereotactic body radiation therapy an attractive option for unresectable liver metastases? A preliminary report from a phase 2 trial. Int J Radiat Oncol Biol Phys 86: 336-342, 2013.

16 Scorsetti M, Comito T, Cozzi L, Clerici E, Tozzi A, Franzese C, Navarria P, Fogliata A, Tomatis S, D'Agostino G, Iftode C, Mancosu P, Ceriani R and Torzilli G: The challenge of inoperable hepatocellular carcinoma(HCC): results of a singleinstitutional experience on stereotactic body radiation therapy(SBRT). J Cancer Res Clin Oncol 141: 1301-1309, 2015.

17 Kopek N, Holt MI, Hansen AT and Høyer M: Stereotactic body radiotherapy for unresectable cholangiocarcinoma. Radiother Oncol 94: 47-52, 2010.

18 Ahmed KA, Caudell JJ, El-Haddad G, Berglund AE, Welsh EA, Yue B, Hoffe SE, Naghavi AO, Abuodeh YA, Frakes JM, Eschrich SA and Torres-Roca JF: Radiosensitivity Differences Between Liver Metastases Based on Primary Histology Suggest Implications for Clinical Outcomes After Stereotactic Body Radiation Therapy. Int J Radiat Oncol Biol Phys 95: 1399-1304, 2016.

19 Ohri N, Jackson A, Mendez Romero A, Miften M, Ten Haken RK, Dawson LA, Grimm J, Yorke ED and Tome WA: Local control following stereotactic body radiotherapy for liver tumors: a preliminary report of the AAPM Working Group for SBRT. Int J Radiat Oncol Biol Phys 90: S52, 2014.

20 Scorsetti M, Comito T, Tozzi A, Navarria P, Fogliata A, Clerici E, Mancosu P, Reggiori G, Rimassa L, Torzilli G, Tomatis S, Santoro A and Cozzi L: Final results of a phase II trial for stereotactic body radiation therapy for patients with inoperable liver metastases from colorectal cancer. J Cancer Res Clin Oncol 141: 543-553, 2015.

21 Vautravers-Dewas C, Dewas S, Bonodeau F, Adenis A, Lacornerie T, Penel N, Lartigau E and Mirabel X : Image-guided robotic stereotactic body radiation therapy for liver metastases: is there a dose response relationship? Int J Radiat Oncol Biol Phys 81: 39-47, 2011

22 Takeda A, Sanuki N, Tsurugai Y, Oku Y and Aoki Y: Stereotactic body radiotherapy for patients with oligometastases from colorectal cancer: risk adapted dose prescription with a maximum dose of 83100 Gy in five fractions. J Radiat Res 57: 400-405, 2016.
23 Takeda A, Kunieda E, Ohashi T, Aoki Y, Koike N and Takeda T: Stereotactic body radiotherapy (SBRT) for oligometastatic lung tumors from colorectal cancer and other primary cancers in comparison with primary lung cancer. Radiother Oncol 101: 255$259,2011$.

24 Oku Y, Takeda A, Kunieda E, Sudo Y, Oooka Y, Aoki Y, Shimouchi Y, Nishina R, Nomura K, Sugiura M and Ohashi T: Analysis of suitable prescribed isodose line fitting to planning target volume in stereotactic body radiotherapy using dynamic conformal multiple arc therapy. Pract Radiat Oncol 2: 46-53, 2012.

25 Oku Y, Takeda A, Sanuki N, Sudo Y, Oooka Y, Aoki Y, Shimouchi Y, Nishina R, Nomura K, Eriguchi T and Kunieda E: Stereotactic ablative body radiation therapy with dynamic conformal multiple arc therapy for liver tumors: optimal isodose line fitting to the planning target volume. Pract Radiat Oncol 4: 7-13, 2014

26 Giraud P, Antoine M, Larrouy A, Milleron B, Callard P, De Rycke Y, Carette MF, Rosenwald JC, Cosset JM, Housset M and Touboul E: Evaluation of microscopic tumor extension in nonsmall-cell lung cancer for three-dimensional conformal radiotherapy planning. Int J Radiat Oncol Biol Phys 48: 10151024, 2000

27 Lee MT, Kim JJ, Dinniwell R, Brierley J, Lockwood G, Wong R, Cummings B, Ringash J, Tse RV, Knox JJ and Dawson LA: Phase I study of individualized stereotactic body radiotherapy of liver metastases. J Clin Oncol 27: 1585-1591, 2009.

28 Scorsetti M, Clerici E, Navarria P, D'Agostino G, Piergallini L, De Rose F, Ascolese A, Tozzi A, Iftode C, Villa E, Comito T, Franzese C, Mancosu P, Tomatis S and Cozzi L: The role of stereotactic body radiation therapy (SBRT) in the treatment of oligometastatic disease in the elderly. Br J Radiol 88: 20150111 , 2015 . 